This document is confidential and is proprietary to the American Chemical Society and its authors. Do not copy or disclose without written permission. If you have received this item in error, notify the sender and delete all copies.

\title{
Dynamics of membrane proteins within synthetic polymer membranes with large hydrophobic mismatch
}

\begin{tabular}{|r|l|}
\hline Journal: & Nano Letters \\
\hline Manuscript ID: & nl-2015-00699v.R1 \\
\hline Manuscript Type: & Communication \\
\hline Date Submitted by the Author: & $26-$ May-2015 \\
\hline Complete List of Authors: & $\begin{array}{l}\text { Itel, Fabian; University of Basel, Department of Chemistry } \\
\text { Najer, Adrian; University of Basel, Department of Chemistry } \\
\text { Palivan, Cornelia; University of Basel, Chemistry Department } \\
\text { Meier, Wolfgang; University of Basel, Department of Chemistry }\end{array}$ \\
\hline
\end{tabular}

\section{SCHOLARONE \\ Manuscripts}




\title{
Dynamics of membrane proteins within synthetic
} polymer membranes with large hydrophobic

\author{
mismatch
}

Fabian Itel, Adrian Najer, Cornelia G. Palivan, Wolfgang Meier*

Department of Chemistry, University of Basel, Klingelbergstrasse 80, 4056 Basel, Switzerland

*Corresponding author's contact information:

wolfgang.meier@unibas.ch

fax: +41(0)612673855

phone: +41(0)612673802 


\begin{abstract}
The functioning of biological membrane proteins (MPs) within synthetic block copolymer membranes is an intriguing phenomenon that is believed to offer great potential for applications in life- and medical- sciences and engineering. The question why biological MPs are able to function in this completely artificial environment is still unresolved by any experimental data. Here, we have analyzed the lateral diffusion properties of different sized MPs within poly(dimethylsiloxane) (PDMS)-containing amphiphilic block copolymer membranes of membrane thicknesses between 9 and $13 \mathrm{~nm}$, which results in a hydrophobic mismatch between the membrane thickness and the size of the proteins of $3.3-7.1 \mathrm{~nm}(3.5-5$ times $)$. We show that the high flexibility of PDMS, which provides membrane fluidities similar to phospholipid bilayers, is the key-factor for MP incorporation.
\end{abstract}

\footnotetext{
KEYWORDS: Membrane proteins, lateral diffusion, synthetic membranes, hydrophobic mismatch, fluorescence correlation spectroscopy, amphiphilic block copolymers
} 
Cell membranes are intricate structures composed of self-assembled phospholipids that build into a thin, 2-dimensional viscous sheet. The lipid bilayer, providing a stable but dynamic cell boundary, hosts specific membrane proteins (MPs) to achieve selective membrane transport, which is essential for cellular function. The exceptional transport efficiency of MPs, embedded within membranes, together with the availability of high-resolution analytical techniques, has attracted molecular engineers to design artificial biomimetic membranes for technological applications. ${ }^{1}$ As an improved alternative to phospholipids, amphiphilic block copolymers (BCPs), which imitate the amphiphilic membrane-forming property of lipids, have gained much interest, especially due to the higher mechanical and chemical stability of the corresponding BCP membranes compared to phospholipid bilayers; $;^{2-4}$ this is an essential requirement for advanced and high-throughput technological applications. ${ }^{1}$ Another advantage that is provided by BCP membranes is their ability to form a barrier with reduced permeability to water, ions and neutral molecules compared to natural lipid bilayer membranes. ${ }^{4,5}$ Molecular parameters of the BCP macromolecules, i.e. molecular weight, chemistry of the blocks, etc., determine the properties of the final, self-assembled membrane. ${ }^{6}$ The membrane properties can therefore be tuned by simply changing those molecular parameters to optimize the membrane for a desired technological application. Recently, biomimetic membranes based on BCPs have been successfully used for insertion of sensitive, biological MPs, ${ }^{5,7-19}$ biopores/ionophores, ${ }^{20-22}$ and polysaccharide-based cell receptors. ${ }^{23}$ It has been shown that MPs embedded in these synthetic membranes are able to specifically and efficiently tune the permeability properties to a specific need. Since the function of the MP relies on its molecular stability and structural flexibility, determined by the tertiary and quaternary structure of the protein, the synthetic BCP membrane, analogous to the lipid bilayer, has to provide a supporting matrix to retain the protein's structure. Equally to natural 
phospholipid membranes, the insertion process and alignment of the MP within the membrane is based on burying the hydrophobic amino acid residues in the hydrophobic part of the membrane, while the hydrophilic residues face the aqueous side and/or the hydrophilic part of the membrane. In addition to the insertion process of sensitive MPs, a key parameter for their functionality is the lateral mobility within membranes, a property depending on the flexibility and fluidity of the membrane. ${ }^{24}$ Many cellular processes, such as energy- and signal transduction, sensing, etc., involve several MPs together and rapid continuous mixing within the membrane is essential. $^{25-29}$ Therefore, these synthetic BCP membranes have to fulfill key molecular properties such as hydrophobicity, fluidity and flexibility.

Besides the aforementioned parameters, the large thickness of BCP membranes, which can be $2-10$ times that of phospholipid bilayers, leads to a large mismatch between the membrane thickness and the size of the MP, which is expected to significantly affect the insertion, mobility and functionality of biomolecules. ${ }^{4,5,30}$ Theoretical calculations have indicated that BCP membranes are capable of adjusting their thickness to the size of the membrane inclusion / MP with a hydrophobic mismatch change of $1.3 \mathrm{~nm}(22 \%))^{30}$ This molecular dynamics simulation explained that the BCP chains are able to compress in the vicinity of a MP, and the effect is greater with increasing flexibility of the polymer type. Recent studies have shown that MPs/biopores remained functional in BCP membranes, which were up to 6 times thicker than the height of the MPs/biopores. ${ }^{5,7-9,12-21}$ Hydrophobic mismatches in BCP membranes are therefore extremely high compared to those reported in biological membranes. ${ }^{31}$ Thus, it is remarkable how these synthetic BCP membranes provide an environment, which maintains MP function. Consequently, the challenges of MP insertion and its functionality are high, because of the 
complex scenario of requirements imposed on a synthetic membrane (hydrophobicity and size, flexibility, elasticity, density, etc.).

We recently characterized the lateral diffusion properties of $\mathrm{BCP}$ membranes consisting of poly(2-methyl-2-oxazoline) (PMOXA) and poly(dimethylsiloxane) (PDMS) with different membrane thicknesses $(6-20 \mathrm{~nm})$ and different block architectures (diblock and triblock, PMOXA- $b$-PDMS-(b-PMOXA)) ${ }^{32}$ We observed that the fluidity properties of these synthetic membranes were similar to the diffusion of lipids in phospholipid bilayers. In addition, we showed that interdigitation and entanglement of the BCP chains were the main factors contributing to the decrease in lateral diffusion, whereas the high fluidity and flexibility of PDMS inhibits the reduction in mobility of the macromolecules, as shown for other types of polymers. ${ }^{33}$ Therefore, these synthetic BCP membranes mimic natural lipid bilayers with respect to the membrane fluidity and do not have to be blended with fast diffusing lipids in order to increase their fluidity. ${ }^{34}$ Furthermore, the functional incorporation of a small biopore (gramicidin) with height in the range of the present MPs in BCP giant unilamellar vesicles (BCPGUVs), similar to the GUVs used in this study, was reported very recently. ${ }^{21}$ Gramicidin was successfully inserted in synthetic membranes up to $13 \mathrm{~nm}$ thick, whereas thicker membranes (for example $16.2 \mathrm{~nm}$ ) prevented a functional biopore insertion.

However, it is still unclear how sensitive biological MPs are able to function in a completely synthetic membrane. Therefore, it is important to understand, which molecular parameters of the membrane play key roles in providing an appropriate environment for MPs to allow their insertion and functionality. Until now, no experimentally determined diffusion properties of membrane-reconstituted biological species within synthetic BCP membranes have been reported. Here, we introduce a detailed view on how different sized MPs diffuse within self-assembled 
synthetic BCP membranes with thicknesses of 9.2, 12.1, and $13.4 \mathrm{~nm}$. These large thicknesses induce a significant hydrophobic thickness mismatch ranging from $3.3 \mathrm{~nm}$ to $7.1 \mathrm{~nm}$, compared to hydrophobic mismatches in phospholipid membranes, where the thickness difference is only \pm $1.0 \mathrm{~nm}$. We selected three different PMOXA- $b$-PDMS- $b$-PMOXA triblock copolymers, further referred to as $\mathrm{ABA} 34, \mathrm{ABA} 49$ and $\mathrm{ABA} 63$, as the membrane forming BCPs, where the number is attributed to the number of the PDMS units. Only different molecular weights distinguish these BCPs, which have constant chemical composition (PMOXA and PDMS) and block architecture (triblock, ABA) (Table 1). The following MPs were selected as models: the potassium crystallographically-sited activation channel (KcsA), the bacterial water-selective channel protein AquaporinZ (AqpZ), and the outer membrane protein F (OmpF). An important point is that the conditions for protein insertion were similar to published data where functionality of OmpF and AqpZ have been demonstrated for copolymer membranes of polymersomes with thicknesses of about $10 \mathrm{~nm}$ and higher. ${ }^{5,7,11-13,16,17}$ Furthermore, we were interested in determining the lateral diffusion coefficients of MPs of different lateral dimensions (radius) in order to establish whether molecular parameters, such as flexibility of the membrane, are responsible for MP insertion. Together with membrane thickness, the flexibility of the membrane represents a crucial molecular parameter when MPs have to be inserted in thick synthetic membranes and preserve their functionality.

Table 1. Molecular characteristics of membrane-forming amphiphiles (lipid and triblock copolymers).

\begin{tabular}{cccccccc} 
Membrane & Amphiphile & ID & $\mathrm{M}_{\mathrm{w}}[\mathrm{g} / \mathrm{mol}]$ & $\begin{array}{c}\mathrm{M}_{\mathrm{w}, \text { PDMS }} \\
{[\mathrm{g} / \mathrm{mol}]}\end{array}$ & PDI & $\begin{array}{c}\mathrm{f}_{\text {hydrophilic }} \\
{[\%]}\end{array}$ & $\mathrm{d}[\mathrm{nm}]$ \\
\hline Lipid & POPC & POPC & 770 & - & - & $\sim 35$ & $5.0 \pm 0.4^{*}$ \\
\hline Triblock & $\mathrm{A}_{6} \mathrm{~B}_{34} \mathrm{~A}_{6}$ & ABA34 & 3800 & 2600 & 2.3 & 32 & $9.2 \pm 0.5^{* *}$ \\
& & & \multicolumn{2}{c}{6}
\end{tabular}


copolymers

$$
\mathrm{A}_{7} \mathrm{~B}_{49} \mathrm{~A}_{7}
$$

ABA49

5100

3700

2.1

27

$12.1 \pm 1.0^{* *}$

$\mathrm{A}_{12} \mathrm{~B}_{63} \mathrm{~A}_{12} \quad \mathrm{ABA} 63 \quad 6900$

4700

2.1

32

$13.4 \pm 0.9 * *$

*determined from cryo-TEM. $* *$ from reference ${ }^{32}$.

MPs are known to be able to functionally insert into PDMS-containing ABA membranes, while retaining the tertiary and quaternary structure of the protein, and thus their activity, as reported in several studies..$^{5,7-9,12,13,15-19,35-41}$ Specifically, the functionality of AqpZ and OmpF in such synthetic membranes has been studied extensively, while KcsA was chosen solely because of its smaller size than AqpZ and OmpF in order to determine the effect of the protein radius on lateral diffusion. In addition, gramicidin, a biopore with $2.5 \mathrm{~nm}$ height was successfully inserted, and preserved its functionality in membranes up to $13 \mathrm{~nm}$ thickness. $^{21}$

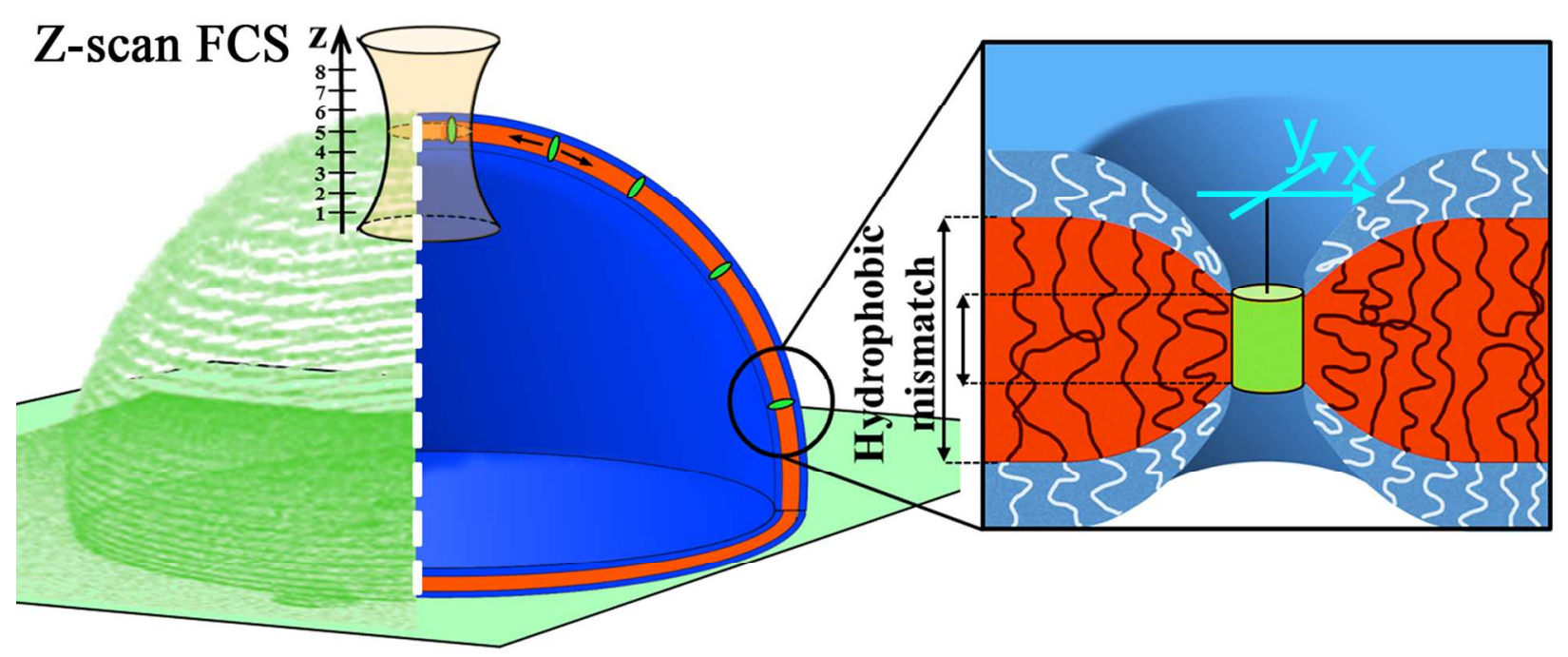

Scheme 1. Schematic representation of the measurement principle and hydrophobic mismatch between the membrane thickness and the MP (represented as green cylinder). Giant unilamellar vesicles self-assembled from BCPs (BCP-GUVs, left) are adsorbed as hemispheres on the glass surface to form stable membranes for precise z-scan FCS measurements. The left half of the BCP-GUV represents a 3-D fluorescence image of the incorporated fluorescent MPs 
and the right half represents the schematic BCP-GUV membrane. Inserted MPs (labeled with a fluorescent dye) are mobile within the synthetic BCP membrane and can diffuse in the 2dimensional plane of the membrane similar to the situation in a lipid bilayer.

As model membranes, we generated BCP-GUVs ( $5-50 \mu \mathrm{m}$ in diameter $)$ with inserted MPs. BCP-GUVs were prepared by the electroformation technique. ${ }^{42}$ In detail, the detergent that stabilized the MPs in aqueous solution was first exchanged with ABA BCPs by the standard vesicle formation method via the film rehydration technique, followed by dialysis and extrusion to yield BCP-stabilized MPs (BCP-MPs). ${ }^{5,38,41}$ We observed strong interaction of detergent molecules with all three ABA membranes, thus purification by long-time dialysis was an important step, as reported previously. ${ }^{38,41}$ In addition, we used a buffer system with low salt concentration ( $1 \mathrm{mM}$ Hepes, $2 \mathrm{mM} \mathrm{NaCl}, \mathrm{pH} 7.4)$ in order to be able to apply an electrical field for the electroformation technique. Second, BCP-MPs were then only partially dried in air on ITO-coated (indium tin oxide) glass plates to form a smooth membrane for subsequent electroformation (see experimental section in supplementary information).

The generated MP-containing BCP-GUVs were adsorbed, and thus immobilized, on plasmatreated glass surfaces resulting in stable hemispheres (Scheme 1). ${ }^{32}$ This immobilization process relies on the interaction between the plasma-treated glass surfaces and the hydrophilic PMOXA block. Due to this relatively strong interaction, which increases with increasing PMOXA block length, the GUVs form hemispheres. The whole area at the bottom of the sphere consists of the BCP membrane providing a large area of interaction. The free-standing membrane on top of the BCP-GUV provides an ideal model membrane, which also avoids any interaction-artifacts with the surface. The lateral diffusion was measured by z-scan fluorescence correlation spectroscopy (z-scan FCS), as reported previously. ${ }^{32,43}$ Z-scan FCS relies on determining a serial set of 

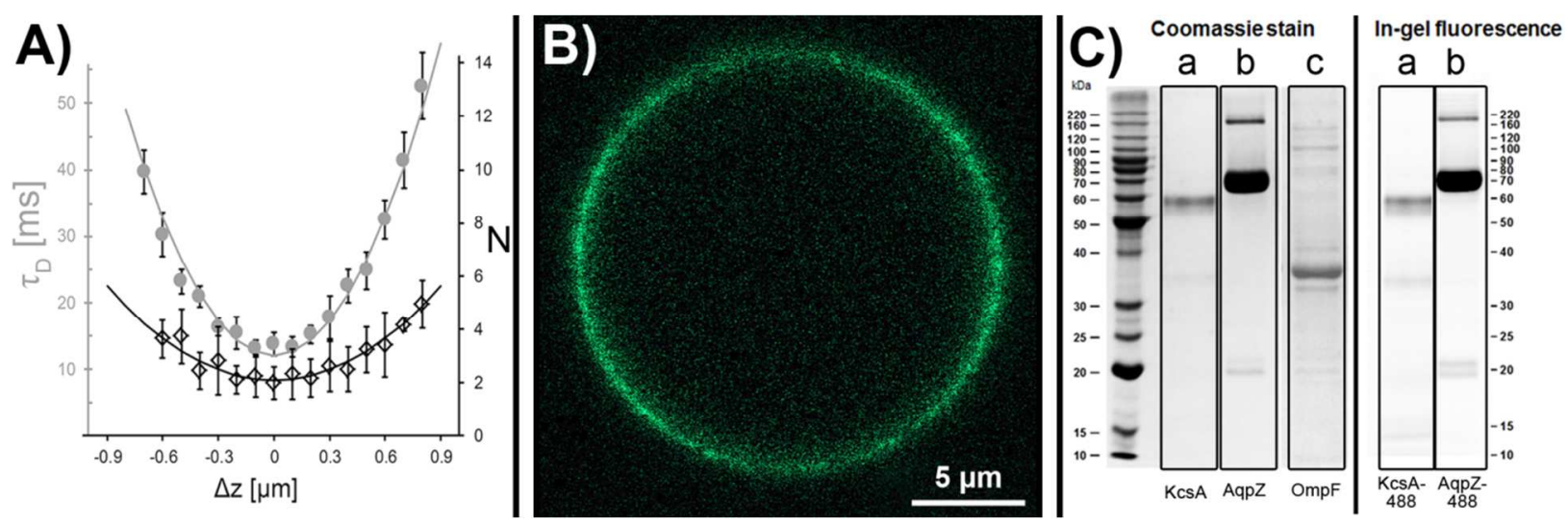

Figure 1. Diffusion- and imaging analysis of BCP-GUVs, and MP purification and labeling.

A) FCS data processing of AqpZ-488 diffusion within ABA49 membrane showing the zdependency of diffusion time $\tau_{D}$ and number of particles $N$. B) LSM image of a BCP-GUV with embedded KcsA, which was labelled with Oregon Green 488. C) SDS-PAGE gel of purified MPs (a: KcsA, b: AqpZ, c: OmpF) and in-gel fluorescence (inverted) of labeled and purified KcsA and AqpZ. The Coomassie stained gel shows the purity of the purified MPs.

For the purpose of obtaining a high signal-to-noise ratio in FCS measurements, fluorescently labeled and unlabeled MPs were mixed at a ratio of 1:10, thus avoiding too many fluorescent MPs in the confocal volume. ${ }^{45}$ MPs were incorporated into ABA membranes at a targeted polymer-to-protein ratio (PoPR) of $50(\mathrm{w} / \mathrm{w})($ Table S2). In fact, the actual PoPR in the final sample is lower because of non-perfect incorporation, which has also been reported for the preparation of lipid-MP vesicles. ${ }^{46}$ We calculated incorporation efficiencies in the order of $5 \%$ (Table S2). The low incorporation efficiency observed in this study within BCP-GUVs, however, 
might still be caused by the drying process of the BCP-MPs before the formation of BCP-GUVs because MPs are very sensitive upon drying. Therefore, the partial drying process of the BCPMPs still caused some aggregation (precipitation) of MPs in the membrane. It has to be noted that a too long drying process has to be avoided in any case. Other studies reported drying of lipid-MP vesicles under vacuum for 12 hours, but the activity of reconstituted MPs within lipid membranes could be preserved only by the addition of minimal amounts of sucrose ${ }^{47}$ or ethylene-glycol. ${ }^{25}$ Here, we generated BCP-GUVs without drying the BCP-MP samples under vacuum (see experimental section in SI). The number of BCP-GUVs formed was relatively small compared to BCP-GUVs generated from pure ABA films. By using laser scanning microscopy (LSM), suitable BCP-GUVs (i.e. non-moving, stable hemispheres, $15-25 \mu \mathrm{m}$ diameter $)^{32}$ were selected for z-scan FCS measurements. As shown in Figure 1B, the fluorescence-labeled fraction of MPs is homogeneously distributed within the ABA membrane. However, in some cases we observed BCP-GUVs with inhomogenously distributed fluorescence indicating MP aggregation. These BCP-GUVs were avoided for FCS measurements because the intense peaks (count rate) in the FCS raw data superimpose on the signal of the non-aggregated MPs, and thus produce an additional shoulder in the FCS autocorrelation function (Figure S1).

Figure 2 shows the plot of the diffusion coefficients, which characterize the MP diffusion within triblock copolymer membranes of different membrane thicknesses (Table S1-S3). During the analysis of the FCS autocorrelation functions, we detected traces of free-dye in the BCPGUV membranes originating from MP labeling (Figure S2). As this minimal amount of free dye $(10-20 \%)$, present in the BCP-GUV membrane as a result of the slight hydrophobic character of OG488, ${ }^{48}$ influences the lateral diffusion measurement, we used a two-component fitting model (see materials and methods in supporting information). In order to evaluate whether small 
molecular mass fluorescent dyes diffuse similarly to the labeled MPs within the membranes, we first verified the diffusion of a selected dye as model. Bodipy-630/650 was chosen based on its small molecular weight and its hydrophobic character supporting an easy insertion into the membrane (Figure S3). The diffusion coefficient of Bodipy-630/650 within the ABA49 membrane was 3 times higher than the ABA49 macromolecules themselves $(4.6 \pm 0.5$ and $1.6 \pm$ $0.2 \mu \mathrm{m}^{2} / \mathrm{s}$, respectively). In addition, small molecular weight hydrophobic molecules diffuse freely $\left(t_{0} \approx 0\right)$ within the membrane (Figure S3B) contrary to pure macromolecules, which show a hindered-diffusion character. ${ }^{32}$ In the case of the more hydrophilic dye OG488, which was used for labeling the MPs, the diffusion coefficient of $7.4 \pm 0.9 \mu \mathrm{m}^{2} / \mathrm{s}$ was higher than the diffusion of the hydrophobic dye Bodipy (Figure S4), as expected from their slight differences in molecular weight.

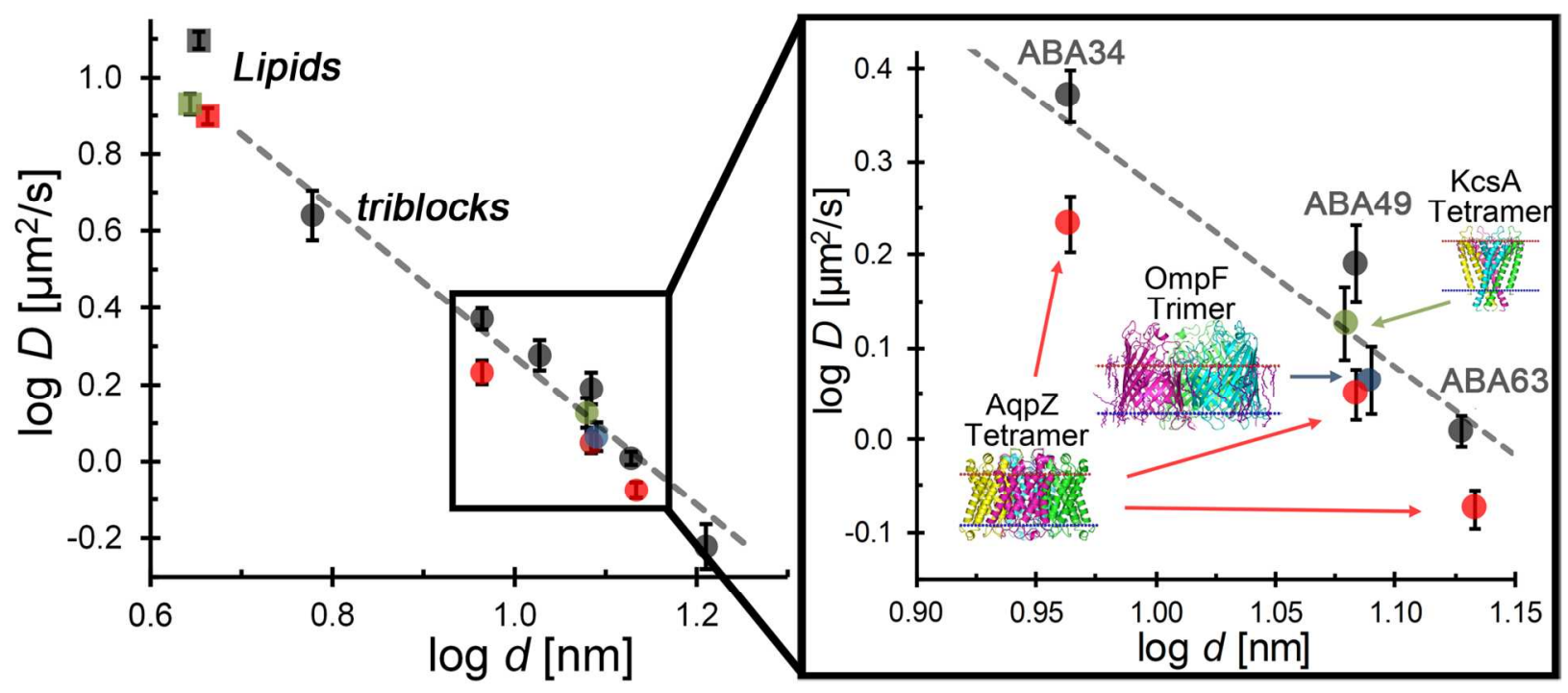

Figure 2. Log-log plots of the diffusion coefficient $D$ relative to membrane thickness $d$. Lipids (squares) and triblock copolymers (circles) are taken as reference membrane systems from ref ${ }^{32}$. The membranes self-assembled from different triblock copolymer have different molecular weights and thus different membrane thicknesses. The dashed line represents the power law 
dependence of the diffusion coefficient in relation to the membrane thickness as $D \sim d^{-1.25}$. The zoomed area shows diffusion coefficients for KcsA (green), AqpZ (red) and OmpF (blue) within the three different ABA membranes (ABA34, ABA49, ABA63) tested in this study.

As reported previously, BCP diffusion within the self-assembled membrane decreases with increasing molecular weight of the BCPs. ${ }^{32}$ The comparison of the lateral diffusion properties of ABAs to those of phospholipids (Figure 2 and Table S1) shows that the diffusion of lipids is around one order of magnitude faster than ABA diffusion (ABA34, ABA49, ABA63). In the case of MP mobility, a fundamental question arises: which are the mechanisms that drive MP insertion into synthetic BCP membranes? MPs are mobile in these membranes, as indicated by FCS experiments (Figure 2).

Hydrophobic mismatch plays an important role when reconstituting MPs into block copolymer membranes of large thicknesses. In this case, the MP height and membrane thickness do not match and the mismatch must be compensated with a structural change in the membrane thickness. Membrane proteins exist with different hydrophobic heights and their interaction with specific types of lipids, for example with specific acyl chain lengths, is of great importance for their function. ${ }^{49}$ However, hydrophobic mismatches occurring in biological membranes are by far less than the ones that may occur in block copolymer membranes. In biological membranes, the thickness differences range between $\pm 1.0 \mathrm{~nm}$. Thus, also negative values exist, where the lipid bilayer has to expand/stretch in the vicinity of a large MP, while a positive mismatch results in membrane thinning. The hydrophobic mismatch $\Delta d$ is calculated as: $\Delta d=d_{\text {hydrophobic }}-$ $d_{M P}$, where $d_{M P}$ is the hydrophobic height of the MPs taken from their crystal structures, and $d$ hydrophobic is the hydrophobic membrane thickness, which was calculated from the measured membrane thickness $d$. Cryo-TEM provided the membrane thickness of the whole polymer and 
not just the hydrophobic part, because the contrast is generated from phase contrast by underfocussing of the objective lens. The phase of the incoming electron beam is shifted at structures with different refractive indices, thus PDMS and PMOXA both provide contrast. As a close approximation, the hydrophobic thickness was calculated from the hydrophilic fraction (fhydrophilic) or the hydrophobic fraction (fhydrophobic) (Table 2). In this way, the hydrophobic thickness of lipid bilayers was obtained relatively accurately from the cryo-TEM images. The POPC lipid bilayer has a membrane thickness of $d=5.0 \pm 0.4 \mathrm{~nm}$ as determined by cryo-TEM

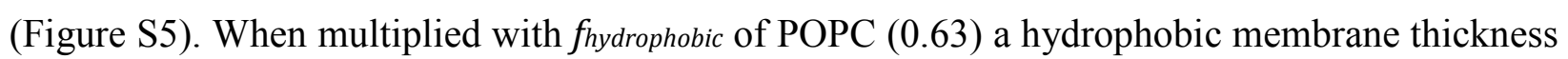
of $3.1 \mathrm{~nm}$ was calculated for a POPC bilayer, which is in agreement with values reported in literature ${ }^{50}$ By using the $f_{\text {hydrophobic }}$ of the BCPs used here, the hydrophobic mismatches were determined to range from 3.3 to $7.1 \mathrm{~nm}$ (Table 2 and Figure S6). Thus, the resulting hydrophobic mismatches in BCP membranes are significantly larger than those in biological membranes.

Table 2. Calculation of the theoretical hydrophobic mismatch $(\Delta d)$ between different membrane hydrophobic thicknesses and MP hydrophobic heights.

\begin{tabular}{|c|c|c|c|c|c|c|}
\hline Membrane & $\begin{array}{c}f_{\text {hydrophobic }} \\
{[\%]}\end{array}$ & $\begin{array}{c}d \\
{[\mathrm{~nm}]}\end{array}$ & $\begin{array}{c}d_{\text {hydrophobic }} \\
{[\mathrm{nm}]}\end{array}$ & $\begin{array}{c}\text { Membrane } \\
\text { protein }\end{array}$ & $\begin{array}{c}d_{M P} \\
{[\mathrm{~nm}]^{*}}\end{array}$ & $\begin{array}{c}\Delta d \\
{[\mathrm{~nm}]}\end{array}$ \\
\hline \multirow{2}{*}{ POPC } & \multirow{2}{*}{0.63} & \multirow{2}{*}{$5.0 \pm 0.4$} & \multirow{2}{*}{$3.1 \pm 0.3$} & $\mathrm{~K} c s \mathrm{~A}$ & $3.5 \pm 0.1$ & $-0.4 \pm 0.4$ \\
\hline & & & & AqpZ & $3.0 \pm 0.1$ & $0.1 \pm 0.4$ \\
\hline ABA34 & 0.68 & $9.2 \pm 0.5$ & $6.3 \pm 0.3$ & AqpZ & $3.0 \pm 0.1$ & $3.3 \pm 0.4$ \\
\hline \multirow{3}{*}{ ABA49 } & \multirow{3}{*}{0.73} & \multirow{3}{*}{$12.1 \pm 1.0$} & \multirow{3}{*}{$8.8 \pm 0.7$} & $\mathrm{~K} c s \mathrm{~A}$ & $3.5 \pm 0.1$ & $5.3 \pm 0.8$ \\
\hline & & & & AqpZ & $3.0 \pm 0.1$ & $5.8 \pm 0.8$ \\
\hline & & & & OmpF & $2.4 \pm 0.1$ & $6.4 \pm 0.8$ \\
\hline ABA63 & 0.75 & $13.4 \pm 0.9$ & $10.1 \pm 0.7$ & AqpZ & $3.0 \pm 0.1$ & $7.1 \pm 0.8$ \\
\hline
\end{tabular}

*from the orientations of proteins in membranes database (OPM). 
Interestingly, despite this large thickness difference, the mobility of the MPs within the membranes is close to the diffusion of the single ABA macromolecules within the membrane itself. The diffusion coefficients of the three different MPs (KcsA, AqpZ, OmpF) within the three different ABA membranes are only slightly lower than pure polymer diffusion. In comparison to MP diffusion in a natural POPC phospholipid bilayer, the difference in diffusion coefficients between the membrane and the MPs are similar when plotted on a logarithmic scale (Figure 2). Moreover, MP diffusion coefficients in a POPE:POPC bilayer reported recently, where one of the studied MPs was also KcsA, are in perfect agreement with our values for $\operatorname{KcsA}^{51}$

The similarity in MP diffusion between membranes that are completely different chemically and structurally (lipid and BCP membrane) indicates that the fluidity of PDMS-containing BCPs supports the insertion of MPs despite the large hydrophobic mismatch. The high flexibility of PDMS as well as the high PDI explains the ability to adapt the polymer chains to the fixed dimensions of the MPs. A possible model for MP insertion is that the protein forces the polymer chains to change their relaxed membrane structure, and to compress in vicinity of the MP, as suggested by molecular simulations. ${ }^{30}$ Conversely, if the flexibility of the polymer chains was lower, which is the case for chemically different BCP types (e.g. PEO-PE, PEO-PBD, PAAPS), ${ }^{52,53}$ MP insertion would be less probable and more difficult to achieve. Indeed, there are only a few examples reported for the insertion of MPs in other types of BCPs. For example, $\mathrm{PEO}_{10}-b-\mathrm{PB}_{12}$ diblock (AB), ${ }^{38}$ DNA- $b-\mathrm{PIB}_{31}(\mathrm{AB})^{54}$ or PIB- $b$-PEO- $b$-PIB triblock $(\mathrm{BAB})^{55} \mathrm{BCP}$ membranes were reported, but only with small block lengths for the hydrophobic domains $(\sim 1000-1700 \mathrm{Da})$, and hydrophobic molecular weights close to those of phospholipids $(\sim 1000$ Da). Such small hydrophobic blocks result in thin membranes, which increase the probability of 
successful MP insertion. Therefore successful insertion of MPs is supported by an appropriate combination of copolymer flexibility and membrane thickness. Indeed, copolymers with lower flexibility than PDMS, such as PB, and thick membranes resulting from the self-assembly of $\mathrm{PB}_{39}-\mathrm{PEO}_{36}$ obstruct MP insertion (data not shown). ${ }^{56}$ In addition, thicker membranes of flexible PMOXA- $b$-PDMS- $b$-PMOXA copolymers (16.2 nm thickness) did not allow functional insertion

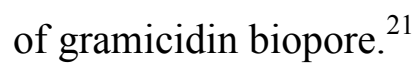

The correct MP insertion was proven by measuring the free lateral diffusion inside the polymer membrane, because it is well known that MPs aggregate very quickly if they are not correctly inserted within the membrane. We also observed samples with precipitated MPs inside GUV membranes (Figure S1), which show that protein precipitation can occur, but usually did not under our experimental conditions. In addition, we very recently reported the activity of a simple biopore, gramicidin, inserted into the same BCP GUVs, which permeabilized the membrane for passage of $\mathrm{H}^{+}, \mathrm{Na}^{+}$and $\mathrm{K}^{+}$ions. ${ }^{21}$ However, it has to be noted that the giant vesicle setup we used here is well suited for the lateral diffusion measurements, but not for activity measurements. Ongoing projects are focused on solving these experimental difficulties, but they do not form part of the present study. The preparation method for BCP-MP vesicles might also be adjusted for other types of block copolymers if their molecular properties support the formation of membranes with thicknesses suitable for protein insertion.

Therefore, we consider that the MPs can influence PDMS-containing BCPs more easily by compressing the polymer macromolecules from the relaxed state (strong-segregation limit, $\mathrm{SSL})^{32}$ to a condensed chain length (membrane thickness compression). This supports the insertion process of biological MPs into PDMS-containing BCP membranes due to the formation 
of a hydrophobic size mismatch, while preserving the tertiary and quaternary structure of the MPs.

Lateral mobility of MPs diffusing within model phospholipid membranes has been determined by several research groups..$^{25,47,51,57}$ Several theoretical models have been proposed to describe the diffusion of MPs in a 2-D membrane, the most famous being the Saffman-Delbrück (SD) model..$^{25,51,57-61}$ The radius of the MPs is one of the factors, which together with membranerelated properties influences the lateral mobility within the membranes as observed within phospholipid bilayers and modeled by the SD-equation (equation 6, SI)..$^{25,51,57,58,62}$ To fulfill their specific function, the MPs used here form quaternary structures by arranging three (trimer) or four (tetramer) single proteins together. As a consequence, the quaternary structure of the proteins determines the size of the diffusing species. We used well-characterized MPs in terms of reported crystal structures, and different sizes with radii of $2.4 \mathrm{~nm}$ (KcsA tetramer), $3.5 \mathrm{~nm}$ (AqpZ tetramer) and $3.8 \mathrm{~nm}(\mathrm{OmpF}$ trimer). The quaternary structure was also preserved in detergent solutions as shown in SDS-PAGE gels (Figure 1C). Therefore, we assume that these multimers are present not only within the POPC lipid membranes, but also within the ABA membranes because they are resistant to SDS detergent solution, and do not disassemble into the monomers. ${ }^{51}$ In addition, the ABA membrane provides a soft environment where the MPs can keep their structure. The SD-model treats the membrane inclusions as cylinders with radius $R$ diffusing freely in a 2-D membrane with thickness $d$ and the membrane viscosity $\left(\eta_{m}\right){ }^{58}$ 


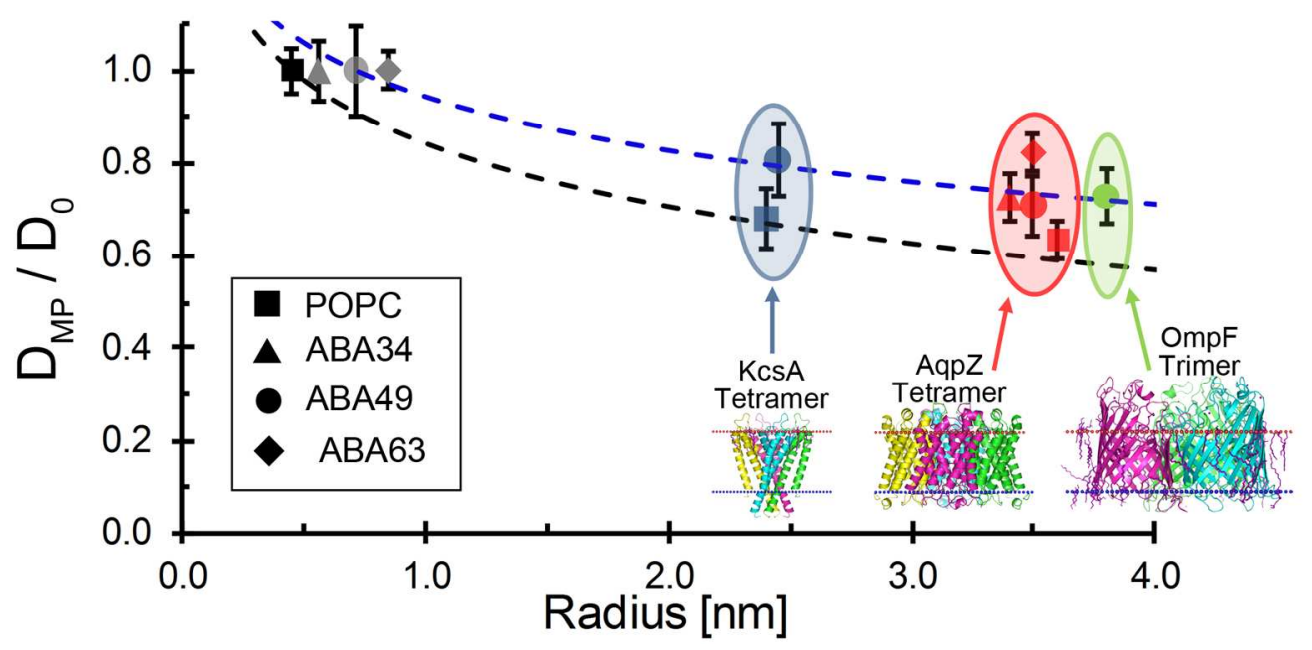

Figure 3. Size-dependent (radius) lateral diffusion of KcsA, AqpZ and OmpF within different membrane systems (natural phospholipids vs. synthetic BCPs). The relative diffusion $\left(D_{\text {prot }} / D_{0}\right)$ of the MPs in comparison to the membrane diffusion shows the similarity between two completely different membrane types. On a relative scale, MP diffusion in the ABA49 membrane (blue dashed line, $\mathrm{R}^{2}=0.94$ ) is very similar to the POPC bilayer (black dashed line, $\left.\mathrm{R}^{2}=0.97\right)$. Diffusion coefficient values are given in Table $\mathrm{S} 1$.

We applied the SD-model to assess its applicability to ABA membranes. The diffusion coefficients of MPs within the ABA49 membrane could be fitted to the SD-equation (Figure 3). In order to see the diffusion coefficients of the MPs within both lipid and synthetic ABA membranes, the results were plotted in terms of relative diffusion (Figure 3), which is defined as the ratio of the MP diffusion $\left(D_{M P}\right)$ to the diffusion of the corresponding membrane $\left(D_{0}\right)$ where the MP is inserted. For completeness, the diffusion coefficients are fitted with the SD-model on the original scale (Figure S7). The resulting membrane viscosity $\left(\eta_{m}\right)$ for the ABA49 membrane yields a value of $126.6 \pm 2.5 \mathrm{mPa} \cdot \mathrm{s}\left(\mathrm{R}^{2}=0.94\right)$, which is four times higher than the membrane viscosity determined for a POPC phospholipid membrane $\left(32.7 \pm 1.2 \mathrm{mPa} \cdot \mathrm{s}, \mathrm{R}^{2}=0.97\right)$. The 
membrane viscosity value obtained for the POPC membrane is in good agreement with the value reported for POPE:POPC phospholipid membrane viscosity $(39.5 \mathrm{mPa} \cdot \mathrm{s}) .^{51}$

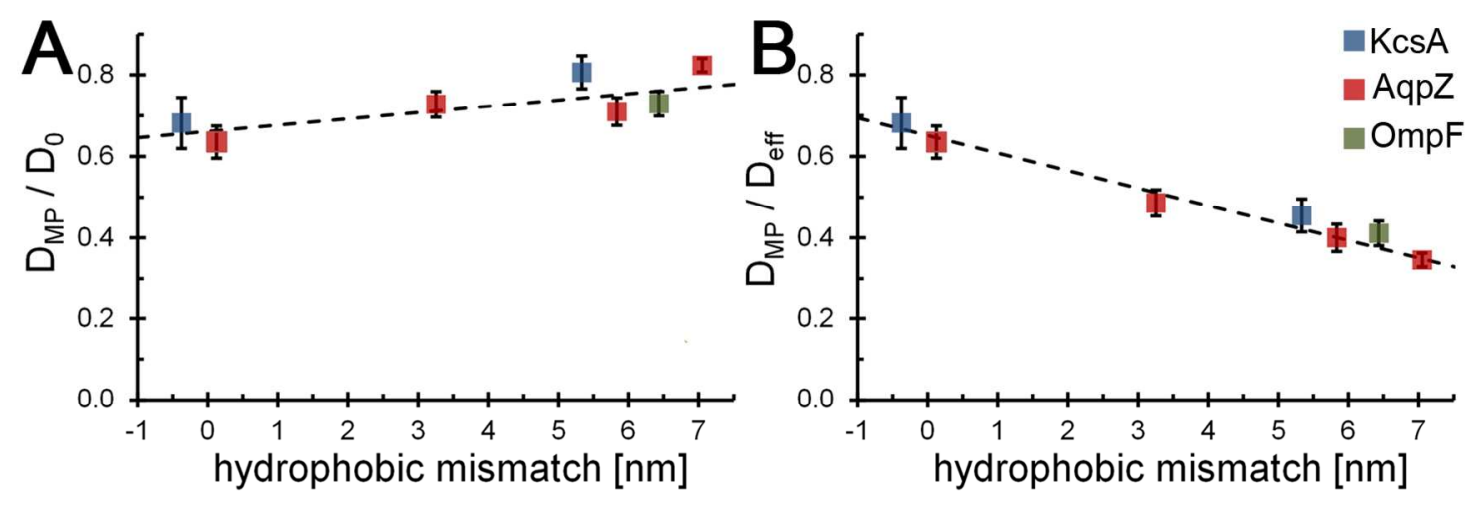

Figure 4. Dependence of the relative diffusion coefficients on the membrane thickness of MPs diffusing within lipid and synthetic ABA membranes. A) The observed relative diffusion coefficient $\left(D_{M P} / D_{0}\right)$ of the MPs increases slightly with increasing membrane thickness. B) The relative, effective diffusion coefficient $\left(D_{M P} / D_{\text {eff }}\right)$ decreases with increasing membrane thickness.

In order to assess the effect of the membrane thickness on the mobility of the MPs, we plotted the relative MP diffusion $\left(D_{M P} / D_{0}\right)$ with respect to the membrane thickness (Figure 4A). Interestingly, it increases slightly with increasing membrane thickness. Due to the formation of domains within the $\mathrm{BCP}$ membranes caused by entanglement and interdigitation of the macromolecules, the measured/observed diffusion of the $\mathrm{BCP}$ macromolecules is in fact a reduced diffusion. This effect is generally described as anomalous diffusion also observed in biological membranes. ${ }^{63-67}$ For example, the presence of domains due to lateral phase separation can lead to multiple diffusion rates in the observation area decreasing the mean value of the measured/observed diffusion coefficient. ${ }^{68-70}$ For BCP membranes, the possibility and strength of interaction of the macromolecules with each other is molecular weight dependent, and thus 
membrane thickness dependent. ${ }^{32}$ Thus, the slight increase in the relative diffusion of the MPs indicates the effect of these domains on their diffusion. The larger these domains, the slower the measured/observed diffusion, and as a result, the relative diffusion of the MPs increases slightly as shown in Figure 4A.

Contrary to the diffusion of the single macromolecules within the ABA membranes, all MPs followed roughly a free-diffusion character $\left(t_{0} \approx 0\right)$, or even a slightly guided-diffusion $\left(t_{0} \leq 0\right)$, based on the analysis of the lateral diffusion coefficients according to the z-scan FCS diffusion law (Table S3). ${ }^{63,71}$ As the hindered diffusion character of the BCP macromolecules within the self-assembled membrane originates from interdigitation and entanglement between the polymer chains, the measured/observed diffusion by FCS is slower than the diffusion of non-entangled chains defined as the effective diffusion $\left(D_{\text {eff }}\right)$. The MPs are not expected to be entrapped in these domains of entangled BCP chains, but rather embedded between them where they are guided through. In order to extract this information, the z-scan FCS law provides a tool for calculating the effective diffusion coefficient $D_{\text {eff }}$ of the ABA membrane. ${ }^{63,72}$ Therefore, $D_{\text {eff }}$ provides a value for the fluidity of the membrane that the MP senses. Due to the measurement of diffusion at different beam waists, i.e. performing the z-scan, the diffusion can be extrapolated to zero beam waist. Whereas the intercept $\left(t_{0}\right)$ yields information about the diffusion characteristics (free-diffusion $t_{0}=0$, hindered diffusion $t_{0}>0$ or guided diffusion $t_{0}<0$ ), the slope is dependent on the size and density of the domains. ${ }^{63}$ For the three different ABA membranes, the slope increases with increasing molecular weight, and thus the decrease in the observed/measured diffusion to $D_{\text {eff }}$ increases with increasing molecular weight as well. On the other hand, the effective diffusion coefficient of the MPs remained roughly the same as the observed diffusion coefficient, which is due to the small $t_{0}$ values obtained for the MPs (Table S3). Because of the 
presence of these domains within the ABA membrane, each MP has to move between them and the larger they are the greater their function in guiding the protein through the membrane. If $D_{\text {eff }}$ is taken as the standard diffusion coefficient of the corresponding membrane, the relative diffusion of the MPs decreases with increasing membrane thickness (Figure 4B). Interestingly, these data suggest that we can observe, on an experimental basis, the effect of the hydrophobic mismatch between the MPs and the large membrane thickness of ABA membrane. Since functional MPs possess a defined size, which is crucial for MP function, the BCP macromolecules have to adapt to the size of the protein. ${ }^{30}$ The effect of adjusting the membrane thickness to the thickness of the MPs was explained by the chain flexibility of the BCP macromolecules. ${ }^{30}$ However, as only two BCP sizes were tested in that molecular dynamics simulation study, there is no information on the maximum possible compressibility. As a consequence, the BCP molecules have to adjust their thickness in close vicinity to the MP. This is more pronounced with larger membrane thickness, and thus the local viscosity increases, and the lateral mobility of the proteins is reduced with increasing membrane thickness. PDMS is well known for its flexibility and low viscosity $\left(T_{g}=-123{ }^{\circ} \mathrm{C}\right),{ }^{73}$ which explains the significant compressibility of the hydrophobic domain around the inserted MPs.

In conclusion, an insight into the local factors characterizing a successful MP insertion process into synthetic BCP membranes requires various essential considerations: i) from fundamental point of view, an understanding of how biomolecules behave in a synthetic environment, and ii) the practical development of new hybrid materials with improved properties and functionality. Biomimetic membranes self-assembled from amphiphilic triblock copolymers composed of PMOXA- $b$-PDMS- $b$-PMOXA offer great potential for use in technological applications, due to their ability to incorporate sensitive biological MPs, and their high chemical 
and mechanical stability. Therefore, this type of block copolymer combines these essential properties. PDMS offers the great advantage of having flexibility and fluidity to entangle and interdigitate to provide stability, while at the same time stretching and compressing in the vicinity of a small biomolecule to preserve its active conformation. A combination of flexible copolymers and appropriate membrane thickness values are necessary for a successful insertion of a MP in synthetic membranes. Flexible copolymers support insertion in thicker membranes than less flexible ones. Thus, we could show that MPs inserted into synthetic BCP membranes that are much thicker than the protein diffuse within the membrane at only an order of magnitude slower than within natural phospholipid membranes. The hydrophobic size mismatch between the membrane thickness and the MP could be observed experimentally by z-scan FCS measurements. This is formed either i) by a contraction of the BCP macromolecules in vicinity of the MP, ii) by the arrangement of smaller BCP chains around the protein whilst the longer chains build up the stable membrane, or iii) by a combination thereof. A thicker membrane induces a stronger compression or a larger domain around the MP. Both processes are thicknessdependent, which reduces the lateral mobility of the MPs within the membrane. Further, the high polydispersity index (PDI) of these block copolymers might also be an essential requirement for successful MP insertion. This study provides both a fundamental basis for the choice of block copolymers to engineer synthetic biomimetic membranes, and supports their implementation into future applications in technology (e.g. membranes for water desalination) and biomedicine (e.g. nanoreactors, artificial organelles). 


\title{
Associated content:
}

Supporting Information Available: Experimental methods, tables with complete experimental data, FCS raw data, and z-scan FCS data for all measured MPs. This material is available free of charge via the Internet at http://pubs.acs.org.

\author{
Author information \\ *(W.M.) Email: wolfgang.meier@unibas.ch; Tel +41 (0)61 26738 02; Fax +41 (0)61 2673855
}

\section{Author Contributions}

The manuscript was written through contributions of all authors. All authors have given approval to the final version of the manuscript.

The authors declare no competing financial interest.

\section{Acknowledgment:}

The Swiss National Science Foundation, the National Centre of Competence in Molecular Systems Engineering (NCCR-MSE) and the University of Basel are acknowledged for financial support. The authors thank Prof. Dr. Sebastian Hiller and Dr. Raphael Böhm from the Biozentrum (University of Basel) for the KcsA plasmid and Christoph Edlinger for providing purified OmpF. We also thank Samuel Lörcher and Sven Kasper for polymer synthesis. The authors thank Dr. B. A. Goodman for editing the manuscript. 


\section{References:}

(1) Shen, Y.-X.; Saboe, P. O.; Sines, I. T.; Erbakan, M.; Kumar, M. J. Membr. Sci. 2014, $454,359-381$.

(2) Palivan, C. G.; Fischer-Onaca, O.; Delcea, M.; Itel, F.; Meier, W. Chem. Soc. Rev. 2012, $41,2800-2823$.

(3) Najer, A.; Wu, D.; Vasquez, D.; Palivan, C. G.; Meier, W. Nanomedicine (Lond) 2013, $8,425-447$.

(4) Discher, B. M.; Won, Y.-Y.; Ege, D. S.; Lee, J. C.; Bates, F. S.; Discher, D. E.;

Hammer, D. A. Science 1999, 284, 1143-1146.

(5) Kumar, M.; Grzelakowski, M.; Zilles, J.; Clark, M.; Meier, W. Proc. Natl. Acad. Sci. USA 2007, 104, 20719-20724.

(6) Itel, F.; Dinu, I. A.; Tanner, P.; Fischer, O.; Palivan, C. G. Nanoreactors for Biomedical Applications. In Handbook of Nanobiomedical Research; Torchilin, V., Ed.; World Scientific, 2014; pp 457-508.

(7) Nardin, C.; Thoeni, S.; Widmer, J.; Winterhalter, M.; Meier, W. Chem. Commun. 2000, $1433-1434$.

(8) Graff, A.; Sauer, M.; Van Gelder, P.; Meier, W. Proc. Natl. Acad. Sci. USA 2002, 99, 5064-5068.

(9) Ranquin, A.; Versées, W.; Meier, W.; Steyaert, J.; Van Gelder, P. Nano Lett. 2005, 5, $2220-2224$.

(10) Choi, H.-J.; Montemagno, C. D. Nano Lett. 2005, 5, 2538-2542.

(11) Nallani, M.; Benito, S.; Onaca, O.; Graff, A.; Lindemann, M.; Winterhalter, M.; Meier, W.; Schwaneberg, U. J. Biotechnol. 2006, 123, 50-59. 
(12) Grzelakowski, M.; Onaca, O.; Rigler, P.; Kumar, M.; Meier, W. Small 2009, 5, 25452548

(13) Graff, A.; Fraysse-Ailhas, C.; Palivan, C. G.; Grzelakowski, M.; Friedrich, T.; Vebert, C.; Gescheidt, G.; Meier, W. Macromol. Chem. Phys. 2010, 211, 229-238.

(14) Dobrunz, D.; Toma, A. C.; Tanner, P.; Pfohl, T.; Palivan, C. G. Langmuir 2012, 28, $15889-15899$.

(15) Wang, H.; Chung, T.-S.; Tong, Y. W.; Jeyaseelan, K.; Armugam, A.; Chen, Z.; Hong, M.; Meier, W. Small 2012, 8, 1185-1190.

(16) Langowska, K.; Palivan, C. G.; Meier, W. Chem. Commun. 2013, 49, 128-130.

(17) Langowska, K.; Kowal, J.; Palivan, C. G.; Meier, W. J. Mater. Chem. B 2014, 2, 46844693.

(18) Grzelakowski, M.; Cherenet, M. F.; Shen, Y.-X.; Kumar, M. J. Membr. Sci. 2015, 479, $223-231$.

(19) Ihle, S.; Onaca, O.; Rigler, P.; Hauer, B.; Rodríguez-Ropero, F.; Fioroni, M.;

Schwaneberg, U. Soft Matter 2011, 7, 532-539.

(20) Sauer, M.; Haefele, T.; Graff, A.; Nardin, C.; Meier, W. Chem. Commun. 2001, 24522453.

(21) Lomora, M.; Garni, M.; Itel, F.; Tanner, P.; Spulber, M.; Palivan, C. G. Biomaterials 2015, 53, 406-414.

(22) Lomora, M.; Itel, F.; Dinu, I. A.; Palivan, C. G. Phys Chem Chem Phys 2015, DOI:10.1039-c4cp05879h.

(23) Najer, A.; Wu, D.; Bieri, A.; Brand, F.; Palivan, C. G.; Beck, H.-P.; Meier, W. ACS Nano 2014, 8, 12560-12571. 
(24) Lee, A. G. BBA-Biomembranes 2004, 1666, 62-87.

(25) Ramadurai, S.; Holt, A.; Krasnikov, V.; van den Bogaart, G.; Killian, J. A.; Poolman, B. J. Am. Chem. Soc. 2009, 131, 12650-12656.

(26) Engelman, D. M. Nature 2005, 438, 578-580.

(27) Edidin, M. Nat. Rev. Mol. Cell Biol. 2003, 4, 414-418.

(28) Vereb, G.; Szöllosi, J.; Matkó, J.; Nagy, P.; Farkas, T.; Vigh, L.; Mátyus, L.; Waldmann, T. A.; Damjanovich, S. Proc. Natl. Acad. Sci. USA 2003, 100, 8053-8058.

(29) Anderson, R. G. W.; Jacobson, K. Science 2002, 296, 1821-1825.

(30) Srinivas, G.; Discher, D. E.; Klein, M. L. Nano Lett. 2005, 5, 2343-2349.

(31) Andersen, O. S.; Koeppe, R. E. Annu. Rev. Biophys. Biomol. Struct. 2007, 36, 107-130.

(32) Itel, F.; Chami, M.; Najer, A.; Lörcher, S.; Wu, D.; Dinu, I. A.; Meier, W. Macromolecules 2014, 47, 7588-7596.

(33) Lee, J. C. M.; Santore, M.; Bates, F. S.; Discher, D. E. Macromolecules 2002, 35, 323326.

(34) Gettel, D. L.; Sanborn, J.; Patel, M. A.; de Hoog, H.-P.; Liedberg, B.; Nallani, M.; Parikh, A. N. J. Am. Chem. Soc. 2014, 136, 10186-10189.

(35) Onaca, O.; Sarkar, P.; Roccatano, D.; Friedrich, T.; Hauer, B.; Grzelakowski, M.; Güven, A.; Fioroni, M.; Schwaneberg, U. Angew. Chem. Int. Ed. 2008, 47, 7029-7031.

(36) Uehlein, N.; Otto, B.; Eilingsfeld, A.; Itel, F.; Meier, W.; Kaldenhoff, R. Sci. Rep. 2012, $2,1-4$.

(37) Nardin, C.; Widmer, J.; Winterhalter, M.; Meier, W. Eur. Phys. J. E. 2001, 4, 403-410.

(38) Kumar, M.; Habel, J. E. O.; Shen, Y.-X.; Meier, W. P.; Walz, T. J. Am. Chem. Soc. 2012, 134, 18631-18637. 
(39) Meier, W.; Nardin, C.; Winterhalter, M. Angew. Chem. Int. Ed. 2000, 39, 4599-4602.

(40) Tanner, P.; Onaca, O.; Balasubramanian, V.; Meier, W.; Palivan, C. G. Chem. Eur. J. 2011, 17, 4552-4560.

(41) Erbakan, M.; Shen, Y.-X.; Grzelakowski, M.; Butler, P. J.; Kumar, M.; Curtis, W. R. PLoS ONE 2014, 9, e86830.

(42) Angelova, M. I.; Dimitrov, D. S. Faraday Discuss. Chem. Soc. 1986, 81, 303-311.

(43) Benda, A.; Beneš, M.; Mareček, V.; Lhotský, A.; Hermens, W. T.; Hof, M. Langmuir 2003, 19, 4120-4126.

(44) Gielen, E.; vandeVen, M.; Margineanu, A.; Dedecker, P.; Auweraer, M. V. D.; Engelborghs, Y.; Hofkens, J.; Ameloot, M. Chem. Phys. Lett. 2009, 469, 110-114.

(45) Kask, P.; Günther, R.; Axhausen, P. Eur. Biophys. J. 1997, 25, 163-169.

(46) Rigaud, J.-L.; Lévy, D. Reconstitution of Membrane Proteins into Liposomes. In Liposomes, Part B; Methods in Enzymology; Elsevier, 2003; pp 65-86.

(47) Doeven, M. K.; Folgering, J. H. A.; Krasnikov, V.; Geertsma, E. R.; van den Bogaart, G.; Poolman, B. Biophys. J. 2005, 88, 1134-1142.

(48) Hughes, L. D.; Rawle, R. J.; Boxer, S. G. PLoS ONE 2014, 9, e87649.

(49) Stillwell, W. An Introduction to Biological Membranes; Elsevier/Academic Press: Amsterdam, Boston, 2013.

(50) Lewis, B. A.; Engelman, D. M. J. Mol. Biol. 1983, 166, 203-210.

(51) Weiß, K.; Neef, A.; Van, Q.; Kramer, S.; Gregor, I.; Enderlein, J. Biophys. J. 2013, 105, $455-462$.

(52) Bermudez, H.; Hammer, D. A.; Discher, D. E. Langmuir 2004, 20, 540-543.

(53) Luo, L.; Eisenberg, A. Langmuir 2001, 17, 6804-6811. 
(54) Cottenye, N.; Syga, M.-I.; Nosov, S.; Müller, A. H. E.; Ploux, L.; Vebert-Nardin, C. Chem. Commun. 2012, 48, 2615-2617.

(55) Muhammad, N.; Dworeck, T.; Fioroni, M.; Schwaneberg, U. J. Nanobiotechnology 2011, 9,8 .

(56) Nehring, R.; Palivan, C. G.; Casse, O.; Tanner, P.; Tüxen, J.; Meier, W. Langmuir 2009, $25,1122-1130$.

(57) Gambin, Y.; Lopez-Esparza, R.; Reffay, M.; Sierecki, E.; Gov, N. S.; Genest, M.;

Hodges, R. S.; Urbach, W. Proc. Natl. Acad. Sci. USA 2006, 103, 2098-2102.

(58) Saffman, P. G.; Delbrück, M. Proc. Natl. Acad. Sci. USA 1975, 72, 3111-3113.

(59) Naji, A.; Levine, A. J.; Pincus, P. A. Biophys. J. 2007, 93, L49-L51.

(60) Petrov, E. P.; Schwille, P. Biophys. J. 2008, 94, L41-L43.

(61) Guigas, G.; Weiss, M. Biophys. J. 2008, 95, L25-L27.

(62) Guigas, G.; Weiss, M. Biophys. J. 2006, 91, 2393-2398.

(63) Wawrezinieck, L.; Rigneault, H.; Marguet, D.; Lenne, P.-F. Biophys. J. 2005, 89, 40294042.

(64) Weiss, M.; Elsner, M.; Kartberg, F.; Nilsson, T. Biophys. J. 2004, 87, 3518-3524.

(65) Schütz, G. J.; Schindler, H.; Schmidt, T. Biophys. J. 1997, 73, 1073-1080.

(66) Ratto, T. V.; Longo, M. L. Langmuir 2003, 19, 1788-1793.

(67) Almeida, P. F. F.; Vaz, W. L. Lateral diffusion in membranes. In Handbook of Biological Physics; Lipowski, R.and Sackmann E., Eds.; Elsevier, New York, NY, 1995; pp 305-357.

(68) Saxton, M. J. Biophys. J. 1989, 56, 615-622.

(69) Saxton, M. J. Biophys. J. 2007, 92, 1178-1191. 
(70) Štefl, M.; Macháň, R.; Hof, M. Z-Scan Fluorescence Correlation Spectroscopy: A Powerful Tool for Determination of Lateral Diffusion in Biological Systems. In Reviews in Fluorescence 2009; Geddes, C. D., Ed.; Reviews in Fluorescence; Springer New York: New York, NY, 2011; pp 321-344.

(71) Heinemann, F.; Vogel, S. K.; Schwille, P. Biophys. J. 2013, 104, 1465-1475.

(72) Humpolíčková, J.; Gielen, E.; Benda, A.; Fagul'ová, V.; Vercammen, J.; vandeVen, M.; Hof, M.; Ameloot, M.; Engelborghs, Y. Biophys. J. 2006, 91, L23-L25.

(73) Yilgor, I.; Yilgor, E. Poly. Bull. 1998, 40, 525-532. 
For TOC Only

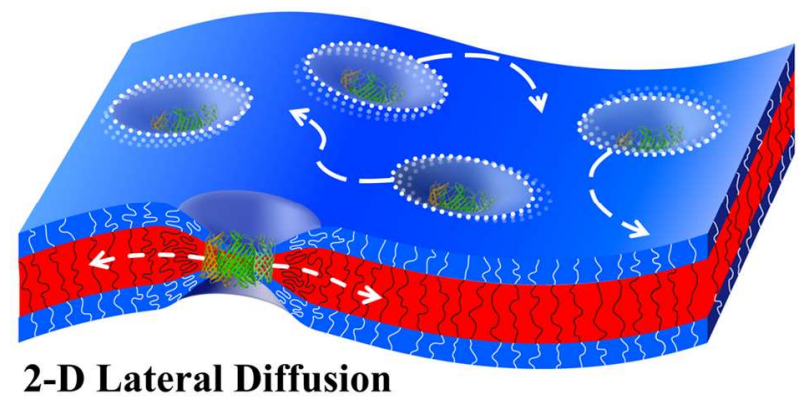

17

18

19

20

21

22

23

24

25

26

27

28

29

30

31

32

33

34

35

36

37

38

39

40

41

42

43

44

45

46

47

48

49

50

51

52

53

54

55

56

57

58

59

60 